\title{
Influencia de los parámetros de procesado y del substrato en la orientación preferente de láminas delgadas de titanato de plomo modificado con calcio preparadas por Sol-Gel
}

\author{
A.GONZÁLEZ, M.L.CALZADA, R.JIMÉNEZ AND J.MENDIOLA \\ Inst. Ciencia de Materiales de Madrid. Cantoblanco. 28049 - Madrid. Spain.
}

\begin{abstract}
En este trabajo se muestran las características texturales de láminas de $\mathrm{Pb}_{0.76} \mathrm{Ca}_{0.24} \mathrm{TiO}_{3}$ preparadas por sol-gel sobre tres substratos diferentes: $\mathrm{Pt} / \mathrm{Ti} / \mathrm{SiO}_{2} / \mathrm{Si}$, Ti $/ \mathrm{Pt} / \mathrm{Ti} / \mathrm{SiO}_{2} / \mathrm{Si}$ y Pt $/ \mathrm{TiO}_{2} / \mathrm{SiO}_{2} / \mathrm{Si}$. Las láminas se depositaron mediante la técnica de centrifugación de la solución precursora, con una concentración muy baja, sobre el substrato, y su posterior secado a $350^{\circ} \mathrm{C}$ y cristalización a $650^{\circ} \mathrm{C}$, utilizando una velocidad de caldeo de $\sim 30.4 \mathrm{~s}^{\circ} \mathrm{C} / \mathrm{s}$. Cada capa de ferroeléctrico obtenida después de este proceso tenía un espesor de $\sim 75 \mathrm{~nm}$. De esta forma se depositaron cuatro capas sucesivas de ferroeléctrico hasta alcanzar un espesor de la lámina de $\sim 300 \mathrm{~nm}$. La textura de las láminas de $\mathrm{Pb}_{0.76} \mathrm{Ca}_{0.24} \mathrm{TiO}_{3}$ sobre los distintos substratos se estudió mediante difracción de rayos X. La fases cristalinas existentes sobre la superficie de cada uno de los substratos se identificaron mediante difracción de rayos $\mathrm{X}$ con ángulo rasante. La lámina de $\mathrm{Pb}_{0.76} \mathrm{Ca}_{0.24} \mathrm{TiO}_{3}$ depositada sobre $\mathrm{Ti} / \mathrm{Pt} / \mathrm{Ti} / \mathrm{SiO} / \mathrm{Si}$ presentó una orientación preferente en el eje 111 de la perovskita. En esta muestra se midió un coeficiente piroeléctrico espontáneo de $\gamma \sim-$ $6.49 \times 10^{-9} \mathrm{Ccm}^{-2} \mathrm{~K}^{-1}$.
\end{abstract}

Palabras clave: sol-gel, lámina delgada, perovskita, textura.

Effect of processing parameters and substrate on the preferred orientation of Sol-Gel calcium modified lead titanate thin films

In this work the characteristics of the texture of sol-gel $\mathrm{Pb}_{0.76} \mathrm{Ca}_{0.24} \mathrm{TiO}_{3}$ deposited onto three different substrates: $\mathrm{Pt} / \mathrm{Ti} / \mathrm{SiO}_{2} / \mathrm{Si}, \mathrm{Ti} / \mathrm{Pt} / \mathrm{Ti} / \mathrm{SiO}_{2} / \mathrm{Si}$ y Pt $/ \mathrm{TiO}_{2} / \mathrm{SiO}_{2} / \mathrm{Si}$, are studied. The films were prepared by spin-coating the precursor solution, with a low concentration, onto the substrates and by further drying at $350^{\circ} \mathrm{C}$ and crystallisation at $650^{\circ} \mathrm{C}$, with a heating rate of $\sim 30.4 \mathrm{~s}^{\circ} \mathrm{C} / \mathrm{s}$. Each of the deposited layers had a thickness of $\sim 75 \mathrm{~nm}$. Four successive layers were deposited to obtain a film thickness of $\sim 300 \mathrm{~nm}$. Texture of the $\mathrm{Pb}_{0.76} \mathrm{Ca}_{0.24} \mathrm{TiO}_{3}$ films on the different substrates was studied by means of X-ray diffraction. The crystalline phases present at the substrates surfaces were identify by means of grazing incidence X-ray diffraction. The $\mathrm{Pb}_{0.76} \mathrm{Ca}_{0.24} \mathrm{TiO}_{3}$ film on the $\mathrm{Ti} / \mathrm{Pt} / \mathrm{Ti} / \mathrm{SiO}_{2} / \mathrm{Si}$ substrate developed a $<111>$ preferred orientation. A spontaneous pyroelectric coefficient of $\gamma \sim-6.49 \times 10^{-9} \mathrm{Ccm}^{-2} \mathrm{~K}^{-1}$ was measured in this sample.

Keywords: sol-gel, thin film, perovskite, texture.

\section{INTRODUCCION}

Entre los distintos procedimientos de preparación de lámina delgada, se encuentra el método sol-gel. Este se engloba dentro de las técnicas químicas de depósito de lámina y presenta indudables ventajas frente a otras técnicas de depósito como son su bajo coste y el alto control estequiométrico y alta reactividad del sistema químico depositado (1). Sin embargo, el solgel se ha venido considerando como una técnica de depósito poco apropiada para la obtención de películas orientadas.

En el caso de las láminas ferroeléctricas basadas en el titanato de plomo, la obtención de depósitos con un alto grado de orientación en la dirección polar de la perovskita, aporta al material un alto valor añadido ya que permite su utilización directa en micro-sensores piroeléctricos y en sistemas microelectro-mecánicos (2), sin necesidad de una polarización previa del material con un campo eléctrico.

La forma de nucleación y crecimiento sobre el substrato de la fase cristalina a partir del depósito amorfo obtenido por solgel, son fundamentales en el crecimiento orientado de la película (3). Una nucleación heterogénea facilita un crecimiento orientado de la película. Ahora bien, este tipo de nucleación se puede inducir, bien a través del substrato o superficie sobre la que la fase cristalina empieza a nuclear (4) y/o, en el caso del depósito por sol-gel, mediante las condiciones de procesado de la lámina (3).

En este trabajo se estudian las condiciones del proceso de depósito por sol-gel que inducen crecimientos orientados de láminas delgadas y el efecto de la superficie sobre la que ésta nuclea, para una composición ferroeléctrica de titanato de plomo modificado, de interés en dispositivos piroeléctricos (5).

\section{EXPERIMENTAL}

Se han preparado láminas delgadas de $\mathrm{Pb}_{0.76} \mathrm{Ca}_{0.24} \mathrm{TiO}_{3}$ mediante el depósito por centrifugación de una solución sintetizada por sol-gel (6) con concentración $0.3 \mathrm{M}$, referida a la perovskita de $\mathrm{Pb}_{0.76} \mathrm{Ca}_{0.24} \mathrm{TiO}_{3}$. A esta solución se le incorporó un $10 \%$ molar en exceso de $\mathrm{PbO}$, para compensar las pérdidas de este compuesto que se van a producir durante el tratamiento térmico de cristalización.

Los substratos sobre los que se realizaron los depósitos fueron substratos de silicio (100) recubiertos de platino con textu- 
ra $<111>$, que además tenían finos depósitos de $\mathrm{Ti}$ o $\mathrm{TiO}_{2}$. Tanto el Pt como el Ti o $\mathrm{TiO}_{2}$ se depositaron sobre el Si mediante pulverización catódica. Los tipos de substratos que se utilizaron para depositar la lámina ferroeléctrica sobre ellos fueron: $\mathrm{Pt} / \mathrm{Ti} / \mathrm{SiO}_{2} / \mathrm{Si}, \mathrm{Ti} / \mathrm{Pt} / \mathrm{Ti} / \mathrm{SiO}_{2} / \mathrm{Si}$ y $\mathrm{Pt} / \mathrm{TiO}_{2} / \mathrm{SiO}_{2} / \mathrm{Si}$. En todos los casos la capa de $\mathrm{SiO}_{2}$ se formó por la oxidación espontánea de la superficie del $\mathrm{Si}$ en contacto con el aire. A las láminas de $\mathrm{Pb}_{0.76} \mathrm{Ca}_{0.24} \mathrm{TiO}_{3}$ depositadas sobre los substratos anteriores las denominaremos $\mathrm{A}, \mathrm{B}$ y $\mathrm{C}$, respectivamente.

El depósito de la solución sobre estos substratos se hizo a $2000 \mathrm{rpm} / 45 \mathrm{~s}$, secando a continuación la lámina a $350^{\circ} \mathrm{C} / 60 \mathrm{~s}$ y cristalizando en aire a $650^{\circ} \mathrm{C} / 50 \mathrm{~s}$, con una velocidad de caldeo de $\sim 30^{\circ} \mathrm{C} / \mathrm{s}$. Este proceso se realizó cuatro veces, para obtener láminas cristalinas con un espesor medio de $\sim 300 \mathrm{~nm}$.

El estudio de la textura de las láminas cristalinas se llevó a cabo mediante Difracción de Rayos X (DRX), utilizando la geometría $\theta-2 \theta$. El equipo utilizado para realizar estas medidas fue un difractómetro de polvo Siemens D500 con un ánodo
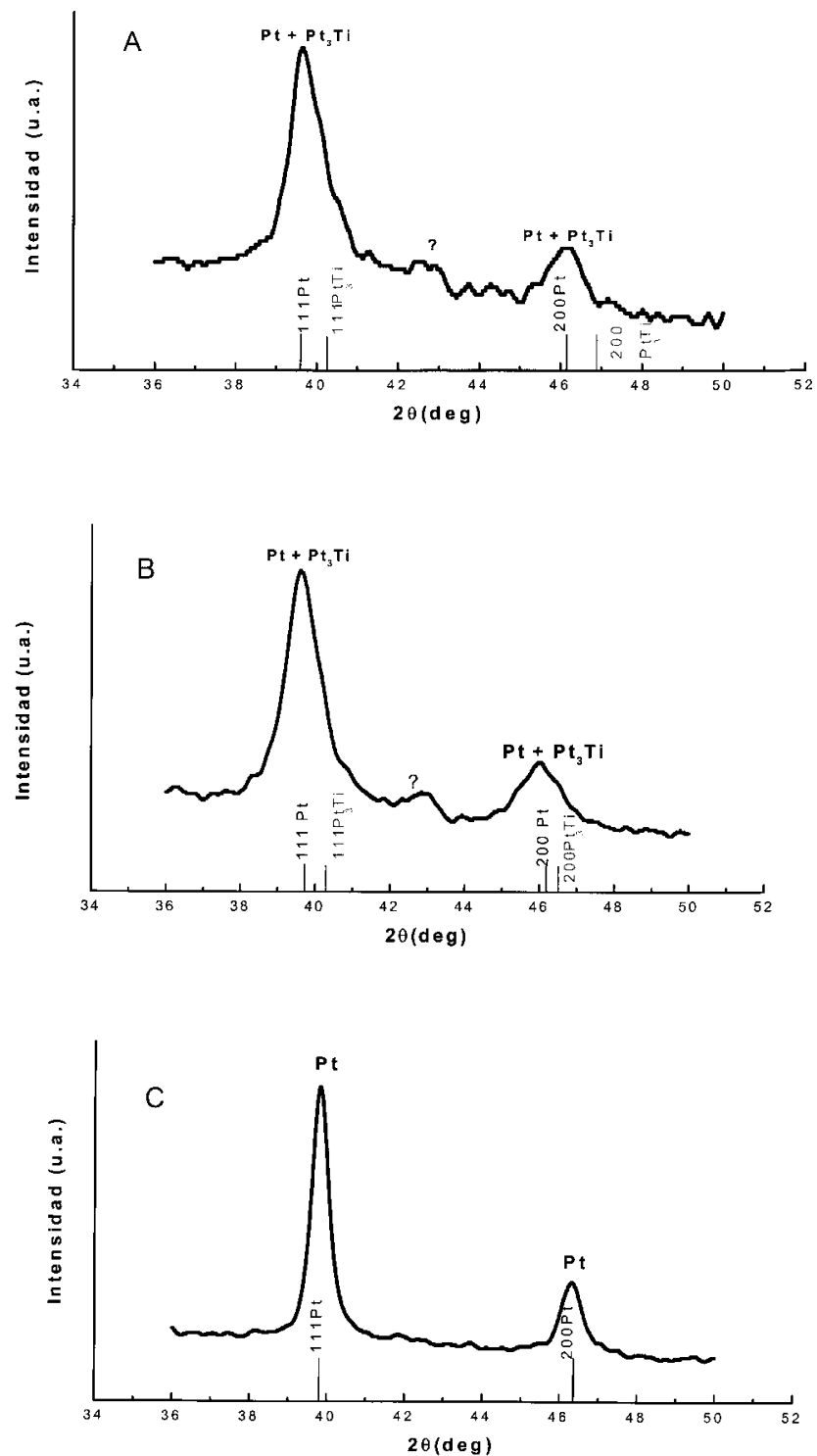

Figura 1: Picos de difracción 001/100, 101/110 y 111 de la perovskita de $\mathrm{Pb}_{0.76} \mathrm{Ca}_{0.24} \mathrm{TiO}_{3}$ resultantes de la deconvolución de los picos medidos experimentalmente para la lámina depositada sobre: a) $\mathrm{Pt} / \mathrm{Ti} / \mathrm{SiO}_{2} / \mathrm{Si}$, b) $\mathrm{Ti} / \mathrm{Pt} / \mathrm{Ti} / \mathrm{SiO}_{2} / \mathrm{Si} \mathrm{y} \mathrm{c)} \mathrm{Pt} / \mathrm{TiO}_{2} / \mathrm{SiO}_{2} / \mathrm{Si}$ de cobre operando a $40 \mathrm{kV}$ y $25 \mathrm{~mA}$. Se realizaron medidas en los intervalos de $2 \theta$ en que aparecían los picos 001/100, 101/110, 111 y 002/200 de la perovskita de $\mathrm{Pb}_{0.76} \mathrm{Ca}_{0.24} \mathrm{TiO}_{3}$ (fichero JCPDS-ICDD \#39-1336). El solapamiento del pico 111 de esta perovskita y el del electrodo de Pt (fichero JCPDSICDD \#4-802) se resolvió utilizando un desfase en $\theta$ de entre $5^{\circ}$ y $10^{\circ}$ respecto al máximo en $2 \theta$ del pico 111 del Pt. Estas condiciones experimentales permitieron separar estos dos picos, aprovechando la fuerte orientación preferente en $<111>$ del Pt, comparada con la misma reflexión de la perovskita, también en su eje 111. El ajuste y separación de picos se realizó mediante un programa del difractómetro que ajustaba los perfiles registrados a una función pseudo-voigt 2.

Las posibles fases cristalinas existentes en la superficie de los substratos sobre la que se deposita la lámina ferroeléctrica se analizaron mediante difracción de rayos $\mathrm{X}$ con ángulo rasante (GIXRD, del inglés grazing incidence $X$-ray diffraction), utilizando un ángulo de incidencia $\alpha=2^{\circ}$.
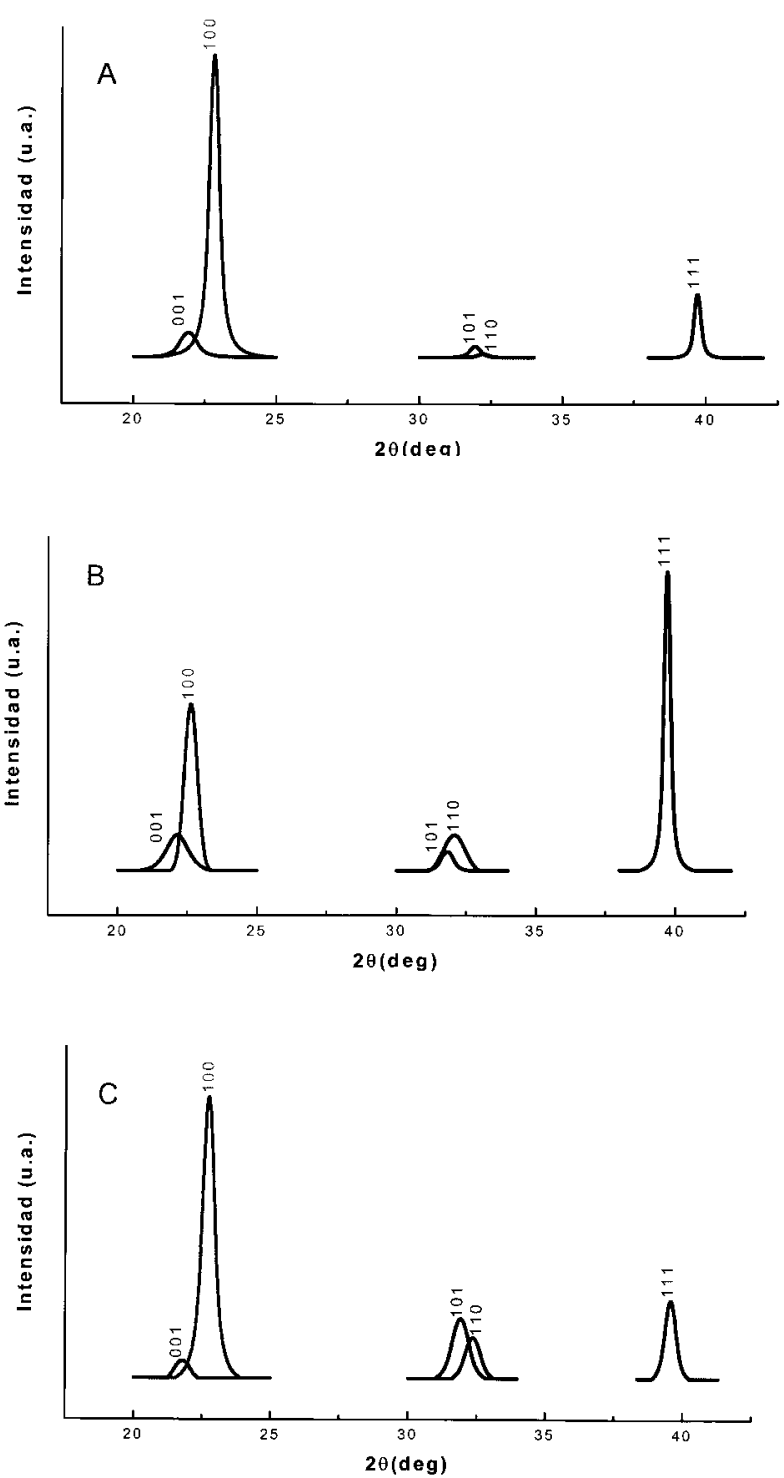

Figura 2: Fases cristalinas detectadas mediante difracción de rayos $\mathrm{X}$ con ángulo rasante $\left(\alpha=2^{\circ}\right)$, sobre la superficie de los substratos: a) $\mathrm{Pt} / \mathrm{Ti} / \mathrm{SiO}_{2} / \mathrm{Si}$, b) $\mathrm{Ti} / \mathrm{Pt} / \mathrm{Ti} / \mathrm{SiO}_{2} / \mathrm{Si}$ y c) $\mathrm{Pt} / \mathrm{TiO}_{2} / \mathrm{SiO}_{2} / \mathrm{Si}$. 
Los coeficientes piroeléctricos espontáneos, $\gamma$, de las láminas de $\mathrm{Pb}_{0.76} \mathrm{Ca}_{0.24} \mathrm{TiO}_{3}$ obtenidas sobre los distintos substratos, se dedujeron de las medidas de las corrientes piroeléctricas obtenidas cuando se aplicaba a las muestras una onda térmica triangular de $\pm 1.5^{\circ} \mathrm{C}$ y $5.10^{-3} \mathrm{~Hz}$, con lo que se conseguía un gradiente efectivo de $\sim 0.03{ }^{\circ} \mathrm{C} / \mathrm{s}(7)$.

\section{RESULTADOS Y DISCUSIÓN}

Las Figuras 2a, 2b y 2c muestran las reflexiones obtenidas en las láminas $\mathrm{A}, \mathrm{B}$ y $\mathrm{C}$, después del ajuste de los picos experimentales medidos mediante DRX. Estos resultados evidencian que en todos los casos las láminas están texturadas. Sin embargo, la orientación preferente predominante en cada una de las muestras es diferente. Las muestras A y C presentan una orientación preferente en la dirección a de la perovskita (paralela al substrato), mientras que la muestra B la presenta según el eje [111], de modo que el eje polar [001] tiene una componente normal a la lámina. Las intensidades de los picos (calculadas con respecto al pico más alto, al que se le da el valor de 100) comparadas con las que se dan en el fichero JCPDSICDD \#39-1336 para el $\mathrm{Pb}_{0.76} \mathrm{Ca}_{0.24} \mathrm{TiO}_{3}$ (en el que la perovskita está orientada al azar) se muestran en la Tabla I. En esta Tabla se indican también los coeficientes piroeléctricos espontáneos medidos en cada tipo de lámina. Como se puede observar, todas las láminas tienen piroelectricidad lo que indica que su textura proporciona una componente en la dirección $c$ normal al plano de la lámina. En el caso de la lámina B, esta componente es fácil de deducir de su orientación preferente a lo largo de $<111>$. Sin embargo, la orientación preferente a lo largo de $<100>$ de las láminas A y C no puede justificar los coeficientes piroeléctricos espontáneos medidos en estas muestras, aunque sí las $<001>$ y $<111>$, de modo que si comparamos las intensidades $\mathrm{I}_{001}$ e $\mathrm{I}_{111}$ de estas muestras con la $\mathrm{I}_{101}$ (que en una perovskita orientada al azar es el pico de más intensidad, ver Tabla I), podemos comprobar que estas orientaciones también aparecen potenciadas, y que por tanto podrían contribuir a la polarización espontánea de estas láminas.

Los factores que los autores de este trabajo consideran que podrían contribuir a la polarización espontánea de estas láminas son tres:

La superficie del sustrato sobre la que se deposita la lámina ferroeléctrica.

El espesor de cada una de las capas de $\mathrm{Pb}_{0.76} \mathrm{Ca}_{0.24} \mathrm{TiO}_{3}$ depositadas y cristalizadas.

Las tensiones desarrolladas durante el depósito y cristalización de las láminas.

El estado de las superficies de los distintos substratos se ha medido mediante difracción de rayos $\mathrm{X}$ con ángulo rasante. Se ha hecho un barrido entre valores de $2 \theta$ dónde aparecen los máximos de difracción de fases cristalinas en las que intervienen, $\mathrm{Pt}$, Ti, Ti-O, Pt-Ti y Pt-Ti-O. El intervalo de $2 \theta$ en el que se han encontrado reflexiones se muestra en la Figura 1. Al analizar estos resultados hay que tener siempre presente las limitaciones de la técnica de DRX para el análisis de superficies: sólo se van a detectar cristales no nanométricos desarrollados en un porcentaje superior a $\sim 3 \%$ respecto a la matriz en que estén inmersos. Además, hay que tener en cuenta que las estructuras buscadas tienen parámetros reticulares muy próximos, con lo que es posible que los picos aparezcan solapados.

Si nos fijamos en la Figura 1c (substrato $\mathrm{Pt} / \mathrm{TiO}_{2} / \mathrm{SiO}_{2} / \mathrm{Si}$ ) solamente se registran dos picos que se pueden ajustar a las reflexiones 111 y 200 del Pt. En los substratos analizados en las
TABLA I: CARACTERÍSTICAS TEXTURALES Y COEFICIENTES PIROELÉCTRICOS DE LAS LÁMINAS DE $\mathrm{PB}_{0.76} \mathrm{CA}_{0.24} \mathrm{TIO}_{3}$ DEPOSITADAS SOBRE LOS SUBSTRATOS $\mathrm{Pt} / \mathrm{Ti} / \mathrm{SiO}_{2} / \mathrm{Si}(\mathrm{A}), \mathrm{Ti} / \mathrm{Pt} / \mathrm{Ti} / \mathrm{SiO}_{2} / \mathrm{Si}$ (B) y Pt $/ \mathrm{TiO}_{2} / \mathrm{SiO}_{2} / \mathrm{Si}$ (C).

\begin{tabular}{|c|c|c|c|c|c|c|c|c|}
\hline \multirow{2}{*}{ muestra } & $\mathrm{I}_{001}$ & $\mathrm{I}_{100}$ & $\mathrm{I}_{101}$ & $\mathrm{I}_{110}$ & $\mathrm{I}_{111}$ & \multicolumn{2}{|c|}{$\begin{array}{c}\text { Fases en la superficie } \\
\text { superficie del substrato }\end{array}$} & \multirow{2}{*}{$\gamma\left(\times 10^{-9} \mathrm{Ccm}^{-2} \mathrm{~K}^{-1}\right)^{5}$} \\
\hline $\mathrm{A}^{1}$ & 9 & 100 & 3 & 2 & 36 & $\mathrm{SI}$ & $\mathrm{SI}$ & -4.70 \\
\hline $\mathrm{B}^{2}$ & 11 & 66 & 9 & 10 & 100 & $\mathrm{SI}$ & $\mathrm{SI}$ & -6.49 \\
\hline $\mathrm{C}^{3}$ & 9 & 100 & 23 & 18 & 29 & $\mathrm{SI}$ & NO & -4.76 \\
\hline Teóricas $^{6}$ & 13 & 21 & 100 & 45 & 34 & NO & NO & - \\
\hline
\end{tabular}

sustrato $\mathrm{Pt} / \mathrm{Ti} / \mathrm{SiO}_{2} / \mathrm{Si}$

sustrato $\mathrm{Ti} / \mathrm{Pt} / \mathrm{Ti} / \mathrm{SiO}_{2} / \mathrm{Si}$

sustrato $\mathrm{PtTiO} / \mathrm{SiO}_{2} / \mathrm{Si}$

fases detectadas mediante diftacción de rayos $\mathrm{X}$ con ángulo rasante (GIXRD)

coeficiente piroeléctrico espontáneo (sin polarizar)

valores del fichero de DRX JCPDS-ICDD \#39-1336

Figuras 1 a y 1 b (substratos $\mathrm{Pt} / \mathrm{Ti} / \mathrm{SiO}_{2} / \mathrm{Si}$ y Ti $/ \mathrm{Pt} / \mathrm{Ti} / \mathrm{SiO}_{2} / \mathrm{Si}$, respectivamente), se podrían ajustar también estas reflexiones. Sin embargo, estos picos son muy anchos indicando el posible solapamiento con las reflexiones 111 y 200 de la fase cúbica $\mathrm{Pt}_{3} \mathrm{Ti}$. En estos dos substratos se registra además otro pico de asignación desconocida. La fase de $\mathrm{Pt}_{3} \mathrm{Ti}$ se puede haber formado en ambos substratos por difusión del Ti hacia el $\mathrm{Pt}$ durante su depósito sobre el silicio por pulverización. La posible reacción entre el Ti y el Pt para formar $\mathrm{Pt}_{3} \mathrm{Ti}$, se ve muy desfavorecida en el caso del substrato $\mathrm{Pt} / \mathrm{TiO}_{2} / \mathrm{SiO}_{2} / \mathrm{Si}$, dónde el titanio está ya oxidado.

Las distancias Ti-Ti en el plano (111) de la fase cúbica $\mathrm{Pt}_{3} \mathrm{Ti}$, y de la perovskita, son de $5.52 \AA$ y $5.55 \AA$, respectivamente. Esto indica que el acoplo de ambas estructuras en ese plano está dentro de $\sim 0.5 \%$, lo que podría justificar el crecimiento de la perovskita con orientación preferente en $<111>$. La existencia de esa fase cúbica de $\mathrm{Pt}_{3} \mathrm{Ti}$ induciría la nucleación de cristales de perovskita orientados en $\langle 111\rangle$, siempre que esta primera capa de $\mathrm{Pb}_{0.76} \mathrm{Ca}_{0.24} \mathrm{TiO}_{3}$ fuese lo suficientemente fina como para que la nucleación fuese heterogénea (3). Efectivamente, como se ha indicado en la parte experimental para la obtención de láminas con espesores de $\sim 300 \mathrm{~nm}$, se han depositado y cristalizado cuatro monocapas de ferroeléctrico, lo que indica que el espesor promedio de cada monocapa es de $\sim 75 \mathrm{~nm}$, espesor suficientemente pequeño como para que la perovskita nuclee mayoritariamente de forma heterogénea (8). El depósito de capas tan finas también disminuye el grado de tensiones desarrolladas en las láminas (9), haciendo posible que en este caso sea pequeña la contribución de este factor al grado de orientación de la película.

\section{CONCLUSIONES}

Se han obtenido por sol-gel láminas ferroeléctricas de $\mathrm{Pb}_{0.76} \mathrm{Ca}_{0.24} \mathrm{TiO}_{3}$ con una orientación preferente neta en la dirección polar de la perovskita mediante el depósito y cristalización, con un tratamiento térmico rápido, de capas finas de perovskita sobre substratos de silicio con electrodo de $\mathrm{Pt}$, en los que se han inducido en superficie núcleos de una fase cristalina de $\mathrm{Pt}_{3} \mathrm{Ti}$ que tiene un acoplamiento de los planos $\{111\}$ de $\sim 0.5 \%$ con los planos $\{111\}$ de la perovskita de 
$\mathrm{Pb}_{0.76} \mathrm{Ca}_{0.24} \mathrm{TiO}_{3}$.

AGRADECIMIENTOS.

Este trabajo ha sido financiado por los proyectos BRPRCT98-077 (BRITE-EURAM, U.E.) y MAT98-1068 (CICYT). Los autores agradecen a Rosalía Poyato su colaboración en la realización de las medidas piroeléctricas que se presentan en este artículo.

\section{BIBLIOGRAFÍA}

1. Schwartz, R.W.,"Chemical solution deposition of perovskite thin films".Chem.Mater., 9 2325-2340 (1997).

2. Polla, D.L. and Francis, L.F., "Ferroelectric thin films in micro-electromechani- cal systems applications". MRS Bulletin, 21(7) 59-65 (1996)

3. Scherer, G.W., "Sintering of sol-gel films". J.Sol-Gel Sci.Techn., 8 353-363 (1997).

4. Tani T., Xu Z. And Payne D.A. "Preferred orientations for sol-gel derived PLZT thin layers". Mat.Res.Soc.Symp.Proc., 310 269-274 (1993).

5. Swartz S.L. and Wood V.E. "Ferroelectric thin films". Condensed Matter News, 1(5) 4-13 (1992).

6. Sirera R. and Calzada M.L. "Multicomponent solutions for the deposition of modified lead titanate films". Mater.Res.Bull., 30(1) 11-18 (1995).

7. Jiménez R., Poyato R., Pardo L, Alemany C. y Mendiola J., “Influencia del substrato sobre la textura y propiedades ferroeléctricas de láminas delgadas de PTCa". Bol-Soc.Esp.Ceram.Vidrio 38 [5] 482-486 (1999)

8. Schwartz R.W.; Voigt J.A., Tuttle B.A., Payne D.A., Reichert T.L. and DaSalla R.S., "Comments on the effects of solution precursor characteristics and thermal processing conditions on the crystallization behavior of sol-gel derived lead zirconate titanate thin films". J.Mater.Res., 12(2) 444-456 (1997).

9. Mendiola J., Calzada M.L., Ramos P., Martín M.J. and Agulló-Rueda F. “On the effects of stresses in ferroelectric $(\mathrm{Pb}, \mathrm{Ca}) \mathrm{TiO}_{3}$ thin films". Tin Solid Films, 315 